\title{
Translational Advances in Cancer Prevention Agent Development (TACPAD) Virtual Workshop on Immunomodulatory Agents: Report
}

\author{
Altaf Mohammed', Roderick H. Dashwood ${ }^{2}$, Sally Dickinson ${ }^{3}$, Mary L. Disis ${ }^{4}$, Elizabeth M. Jaffee ${ }^{5}$, Bryon D. \\ Johnson $^{6}$, Samir N. Khleif ${ }^{7}$, Michael N. Pollak ${ }^{8,9}$, Jeffrey Schlom ${ }^{10}$, Robert H. Shoemaker', Sasha E. Stanton ${ }^{11}$, \\ Georg T. Wondrak ${ }^{12}$, Ming You ${ }^{13}$, Hao Zhu ${ }^{14}$, Mark Steven Miller ${ }^{1}$ \\ ${ }^{1}$ Chemopreventive Agent Development Research Group, Division of Cancer Prevention, National Cancer Institute, \\ Rockville, MD, ${ }^{2}$ Center for Epigenetics \& Disease Prevention, Texas A\&M Health, College of Medicine, Houston, TX, ${ }^{3}$ UA \\ Cancer Center, University of Arizona, Tucson, AZ, ${ }^{4} U W$ Medicine Cancer Vaccine Institute, University of Washington, \\ Seattle, WA, ${ }^{5}$ Department of Oncology, Sidney Kimmel Comprehensive Cancer Center, Johns Hopkins University School of \\ Medicine, Baltimore, MD, ${ }^{6}$ Department of Microbiology \& Immunology, Medical College of Wisconsin, Milwaukee, WI, ${ }^{7}$ The \\ Loop Immuno-Oncology Laboratory, Lombardi Comprehensive Cancer Center, Georgetown University Medical Center, \\ Washington, DC, USA, ${ }^{8}$ Lady Davis Institute for Medical Research, Jewish General Hospital, ${ }^{9}$ Departments of Medicine \\ and Oncology, McGill University, Montreal, QC, Canada, ${ }^{10}$ Laboratory of Tumor Immunology and Biology, National Cancer \\ Institute, Bethesda, MD, ${ }^{11}$ Earle A. Chiles Research Institute, Portland, OR, ${ }^{12}$ Department of Pharmacology and Toxicology, \\ College of Pharmacy and UA Cancer Center, University of Arizona, Tucson, AZ, ${ }^{13}$ Center for Cancer Prevention, Houston \\ Methodist Cancer Center, Houston Methodist Research Institute, Houston, TX, ${ }^{14}$ University of Texas Southwestern Medical \\ Center, Dallas, TX, USA
}

The National Cancer Institute (NCl) Division of Cancer Prevention (DCP) convened the "Translational Advances in Cancer Prevention Agent Development (TACPAD) Workshop on Immunomodulatory Agents" as a virtual 2-day workshop on September 13 to 14 , 2021. The main goals of this workshop were to foster the exchange of ideas and potentially new collaborative interactions among leading cancer immunoprevention researchers from basic and clinical research and highlight new and emerging trends in immunoprevention. The workshop included an overview of the mechanistic classes of immunomodulatory agents and three sessions covering the gamut from preclinical to clinical studies. The workshop convened individuals working in immunology and cancer prevention to discuss trends in discovery and development of immunomodulatory agents individually and in combination with other chemopreventive agents or vaccines.

Key Words Immunomodulatory agents, Chemoprevention, Immunoprevention, Preventive vaccines, Clinical trials

\section{INTRODUCTION}

Immunomodulators modify the activity of the immune system. These compounds have the potential to influence the response of the immune system to tumor development during the earliest stages of tumorigenesis. Immunomodulatory agents have shifted therapeutic approaches to cancer treatment where immune suppression has been shown to play a vital role in the resistance of many tumors to standard therapeutic agents. Discovery of novel small molecule immunomodulatory compounds or repurposing of existing immunomodulatory agents may enhance the ability of the immune system to selectively recognize and attack precancerous and cancer cells.

The utility of chemo-immunomodulatory agents to potentiate cancer vaccine efficacy has been increasingly explored not only in the therapeutic setting but also in the prevention setting in recent years. Recent advances in the field have

Received December 6, 2021, Accepted December 14, 2021

Correspondence to Altaf Mohammed, E-mail: altaf.mohammed@nih.gov, https://orcid.org/0000-0003-1058-6909

Mark Steven Miller, E-mail: mark.miller5@nih.gov, https://orcid.org/0000-0003-1191-0977 
also suggested that lower doses of immunomodulatory agents in combination with other preventive agents could be an effective immunoprevention strategy in specific high-risk populations.

The Division of Cancer Prevention of the National Cancer Institute sponsored the Translational Advances in Cancer Prevention Agent Development (TACPAD) Virtual Workshop on Immunomodulatory Agents on September 13 to 14, 2021. The main goals of this workshop were to 1) foster the exchange of ideas and potentially new collaborative interactions among leading cancer immunoprevention researchers from the basic and clinical research areas, 2) highlight new and emerging trends in immunoprevention, and 3) provide an opportunity for new or experienced cancer prevention investigators who want to explore immunoprevention in their research studies.

\section{HIGHLIGHTS OF THE SCIENTIFIC SESSIONS: SURVEY OF MECHANISTIC CLASSES OF IMIMUNOMODULATORY AGENTS}

Dr. Shoemaker's presentation provided an overview of various classes of immunomodulatory agents that can be used for cancer prevention. Immunomodulatory agents span a wide range of mechanistic classes and new agents with novel activities continue to be identified. Perhaps the oldest class are vaccine "adjuvants". The vast majority of marketed vaccine formulations include an adjuvant intended to activate the innate immune system and enhance the adaptive response to the vaccine antigen. Agonist action at toll-like receptors (TLRs) is thought to underly this effect and various adjuvants addressing specific TLRs are under development. Imiquimod, a TLR-7 agonist, has free-standing immune stimulatory activity that is the basis for its use in treatment of genital warts. Topical application of the TLR-4 antagonist resatorvid (TAK242) blocks inflammatory lymphocyte infiltration into the epidermis and is under development for skin cancer prevention.

The recently developed mRNA vaccines for COVID-19 derive an adjuvant effect from the RNA component of the vaccine that can be modulated by varying uracil content or incorporating pseudouracil or other analogs. Newer entries into the immunomodulatory agent class include "molecularly targeted small molecules". Interleukin-15 (IL-15) modulators such as $\mathrm{N}-803$ (IL-15 superagonist) can stimulate activation, proliferation, survival, and cytotoxicity of natural killer (NK) and CD8+ T cells. IL-6 pathway inhibitors such as SC-144 abrogate STAT3 phosphorylation, nuclear translocation, and downstream target gene expression. This pathway is important in regulating immune cell maturation and has direct involvement in the carcinogenic process.

A variety of small molecule inhibitors show immune modulation and cancer prevention in multiple preclinical models. Cytokine modulators such as calcipotriol (a vitamin D3 an- alogue), applied topically, induces thymic stromal lymphopoietin and enhances CD4+ T cell immunity against skin carcinogenesis. The cholesterol pathway modulator avasimibe (AVA) has been shown to potentiate CD8+ effector function and enhance vaccine effects in lung cancer models. "Nonsteroidal anti-inflammatory drugs (NSAIDs)" have been extensively studied as immunomodulatory agents. Celecoxib has distinct preventive activity in preclinical models of colon cancer and the effect is associated with reduction of infiltrating myeloid-derived suppressor cells (MDSCs). Aspirin has been shown to promote dendritic cell activation, inhibit MDSCs, activate $\mathrm{T}$ cell function, and synergize with vaccines to potentiate a T-helper 1 (Th1) immune response. Naproxen promotes immune activation in colon mucosa by activating T cells, DCs, and macrophages in the process. In a recent study using a genetically engineered mouse model of Lynch Syndrome, both aspirin and naproxen enhanced the activity of a multivalent frame-shift peptide vaccine.

"Immune checkpoint modulators", especially PD-1 directed agents, have had great impact on cancer immunotherapy but are viewed by many as lacking an adequate safety profile for use in immunoprevention. Alterations in dose and schedule may allay this concern in the future. The VISTA antagonist CA-170 increases tumor infiltrating CD8+ T cells and enhances effector-memory $T$ cell frequencies and functions of both CD4+ and CD8+ T cells. In a preclinical model of lung cancer, CA-170 combined with a RAS vaccine demonstrated striking cancer preventive efficacy. With the number and scope of available immunomodulating agents, many "combinations" are possible. Careful identification and optimization of combinations may be the key to development of new cancer preventive interventions.

\section{SESSION 1: EARLY DISCOVERY RESEARCH ON IMIMUNOMODULATORY AGENTS FOR CANCER PREVENTION}

Dr. Elizabeth Jaffee's presentation "Intercepting Pancreatic Cancer Development with Oncogene Targeted Immunotherapy", described the promise of early intervention by vaccines and micro-RNAs in delaying disease progression. Pancreatic adenocarcinoma (PDAC) progression is triggered by a complex interaction of genetic mutations, stromal cell interactions, and tumor microenvironmental (TME) signals. Diagnosis usually occurs late in disease progression, making treatment challenging and survival rates extremely poor. PDACs are also considered non-immunogenic, therefore newly emerging immunotherapies that have been successful in other cancers have not significantly progressed PDAC treatment options. Resistance to immunotherapies progresses as normal cells undergo the earliest genetic changes that transform them into early pre-malignant pancreatic intra-epithelial neoplasms ( $\mathrm{Pa}-$ nINs) that eventually accumulate additional genetic changes that lead to early-stage invasive cancer and eventually, to late 
stage PDAC. This transformation process is influenced by both tumor-intrinsic and extrinsic forces within the developing TME.

Accumulating data suggests that immune resistance mechanisms also evolve with this progression, which has led to the hypothesis that early immune intervention can slow or even halt disease progression and improve treatment outcomes. Initial studies examining cancer vaccines targeted to early oncogenic mutations are showing promise in animal models. A listeria-based vaccine engineered to express oncogenic $\mathrm{Kras}^{\mathrm{G} 12 \mathrm{D}}$ combined with and without regulatory $\mathrm{T}$ cell depletion by an anti-CD25 antibody (PC61) and cyclophosphamide showed increased $T$ cell infiltration, decreased disease progression, and increased survival in Kras-driven and p53 mutated genetically engineered mice (Kras ${ }^{\mathrm{G} 2 \mathrm{D} / \mathrm{+}} \operatorname{Trp} 53^{\mathrm{R} 172 \mathrm{H} /+}$; $\mathrm{Pdx}-1-$ Cre $[\mathrm{KPC}]$ mice) with early PanIN lesions but not those with later stage PanINs [1]. Other early oncogenic mutations in PDACs may prove to be therapeutic targets for vaccine development.

Another class of potential therapeutic targets in PDAC progression are micro-RNAs, or miRNAs. Two specific miRNAs, miR-21 and miR-224, demonstrate increased expression throughout premalignant progression of PanINs to PDAC and have previously been reported to interact with cancer-promoting inflammatory pathways and the epithelial-mesenchymal cellular transition process, a key event in tumor progression. Upon investigating these two miRNAs in cell lines derived from KPC mouse models, Dr. Jaffee's laboratory found that miR-21 inhibition decreases cell proliferation, migration, invasion, and also downregulates downstream components of Kras oncogenic signaling pathways. Furthermore, studies showed increased survival of KPC mice after miR-21 knockdown. MiR-224 similarly showed increased expression during PDAC progression and may be a key regulator of fibroblast activation in the tumor microenvironment [2]. There is also emerging evidence that miR-21 expression may be a prognostic indicator in human pancreatic cancer. Further work to examine the role of miR-21 in human pancreatic cancer progression and the functional effects of mi-RNA modulation in sophisticated organoid models will be instrumental to future exploitation of miRNAs as early therapeutic targets in pancreatic cancer.

Dr. Khleif's talk "Immune-Modulation Interference in Early Oncogenesis Event", described the role of oncogenes in malignant transformation, recruitment of an immunosuppressive tumor microenvironment (TME) in the premalignant stages, and the potential for development of cancer immunoprevention strategies to overcome early tumor escape. Oncogenic mutations are crucial drivers of malignant transformation through promoting tumor progression and invasion. Interactions between the host immune system and the tumor play an important role in deciding the fate of the development and the therapeutic outcomes of malignancies. Classically, it has been accepted that oncogene signaling leads to tumor growth through enhanced proliferation and reduced cell death. However, over the past several years increasing evidence have shown that tumor cell-intrinsic oncogenic signaling mediates a crosstalk with the immune system, modulating the TME and in turn affecting the cancer-immunity cycle. The tumor cell-intrinsic oncogenic signaling directly inhibits $T$ cell activation and recruitment as well as enhances the development of immunosuppressive populations into the TME.

Mutations in the Kirsten rat sarcoma viral oncogene (KRAS) is one of the earliest driver mutation events in the oncogenesis process and usually occurs in the pre-malignant phase. They are highly antigenic and efficiently recognized by the $T$ cell receptors, leading to specific $T$ cell activation. Since KRAS mutations are events that occur within the first few cells in the transformation process and since $T$ cell can recognize and should lyse cells containing these mutations, it is hypothesized that oncogenes like KRAS would develop strategies to create a suppressive microenvironment to aid early in tumor escape. Indeed, mutant KRAS leads to the induction of high levels of TGF $\beta$ and IL-10 in tumor cells and promote the generation of suppressive regulatory $T$ cells (Tregs) [3]. Accordingly, Khleif's laboratory established that KRAS mechanistically induces cell transformation not only through the induction of uncontrolled cellular transformation but, critically, also through the creation of a suppressive microenvironment that helps in tumor cell escape, hence supporting oncogenesis, early immune escape, and tumor growth. Mutant KRAS-induced carcinogenesis leads to a very early accumulation of Tregs in the lung tissue prior to tumor formation, further demonstrating a fundamental mechanism for selective early immune escape of cancer cells carrying this early mutation despite the presence of a highly recognizable $T$ cell antigen.

Following the KRAS finding, several other oncogenic signaling pathways have also been found to affect the anti-tumor immune response, such as activated $\beta$-catenin which, through reduction of Chemokine (C-C motif) ligands 4, stops the cancer-immunity cycle at the priming stage [4]; increased cyclooxygenase (COX)-1/2 activity which inhibits $T$ cell priming and recruitment through the production of immunosuppressive prostaglandin E2 [5]; loss of p53 which results in impairment of NK cell recruitment, activation and function [6]; and loss of phosphatase and tensin homolog which affects autophagy, thereby affecting T-cell priming and also results in resistance to T cell killing [7]. Based on their role in oncogenesis and tumor growth, multiple therapeutic strategies targeting these oncogenic pathways have been employed for cancer treatment over the last several decades. However, delineation of the role of these oncogenic signaling in immune escape suggest the potential development of novel approaches for preventive immunotherapeutic therapy.

Dr. Dashwood's presentation "Epigenetic Modifiers for Immunomodulation", described the role of epigenetics in oncogenesis and the use of agents for cancer prevention by 
targeting different readers/writers/erasers that regulate gene expression. Patients with hereditary cancer risk syndromes, such as Lynch Syndrome and Familial Adenomatous Polyposis (FAP), harbor predicted major histocompatibility complex (MHC) neoantigens, arising mainly from missense mutations [8]. Llosa et al. [9] noted that '...mutation-generated neoantigens are truly tumor specific and may not induce immune tolerance to the same extent as self-antigens...ultimately, an altered amino acid due to a coding mutation is only relevant as a tumor neoantigen for $\mathrm{T}$ cells if it can be processed and presented on self-MHC...individual tumors with lower mutational load can nonetheless generate good $\mathrm{T}$ cell neoepitopes if the mutations are appropriately positioned'. A major roadblock to appropriately positioned neoepitopes involves epigenetic silencing of MHC players [10]. This can impede natural immune responses to early neoplastic transformation, in a prevention setting, and antagonize immuno-therapies that seek to boost anti-tumor CD8 T cells via checkpoint blockade.

While screening novel epigenetic 'reader', 'writer' and 'eraser' inhibitors [11,12], single agents and combinations were identified that markedly re-expressed $\mathrm{MHC}$ class I and II components in human colon cancer lines, exhibiting greater sensitivity than normal cell lines under similar testing conditions. Promising drug combinations (JQ1 + suberoylanilide hydroxamic acid, sulforaphane + JQ1, BG45 + dBET6, etc.) and molecular targets for precision medicine were discussed, including $\mathrm{MHC}$ class I components HLA-A, HLA-B, HLA-C, $\beta 2$ microglobulin (B2M), low-molecular mass polypeptide (LMP2) and transporter 1 (TAP1), as well as MHC class II members HLA-DP, HLA-DQ, HLA-DR, and CD47. Mechanistic insights were provided by $3 \mathrm{D}$ cultures, FAP patient organoids, and the Apc-mutant polyposis in rat colon (Pirc) model $[11,12]$.

Drs. Wondrak and Dickinson's talk "Topical Prevention of Skin Photocarcinogenesis: Targeting Toll-like Receptor 4 (TLR4) and Beyond", described their ongoing studies with the TLR4 antagonist resatorvid (TAK-242) for skin cancer photochemoprevention. Cutaneous exposure to solar UV radiation is a causative factor in skin carcinogenesis. The health and economic burden imposed by UV-associated nonmelanoma skin cancer (NMSC) is substantial, creating an urgent need for the development of improved molecular strategies for its prevention and treatment. Previously, it was shown that modulation of cellular stress response pathways, including nuclear factor-erythroid 2 related factor 2 (NRF2) and AP-1, can be harnessed for skin cancer photochemoprevention using natural products (such as bixin and sulforaphane), representing a novel strategy for human skin photoprotection potentially complementing conventional sunscreen-based approaches.

Recently, cumulative preclinical and clinical evidence indicates that pharmacological modulation of immunoregulatory pathways might hold great promise by leveraging the cutaneous anti-tumor immune response for the prevention of NMSC. As part of this strategy, they have explored the involvement of cutaneous TLR4, an emerging key factor underlying the detrimental effects of immune-dysregulation in response to acute and chronic UV exposure, as a potential molecular target for prevention of NMSC. Direct and indirect TLR4 activation, upstream of inflammatory signaling, is elicited by a variety of stimuli, including pathogen-associated molecular patterns (such as lipopolysaccharide) and damage-associated molecular patterns (such as high mobility group box 1 [HMGB1]) that are formed upon exposure to environmental stressors, such as solar UV.

Targeted molecular interventions that positively or negatively modulate TLR4 signaling have shown promise in translational investigations that may benefit skin cancer patients in the near future, as substantiated by their ongoing preclinical and clinical research. Concordantly, they have reported that programmed death ligand-1 (PD-L1), an immune checkpoint family member, is strongly expressed in a significant number of high-risk cutaneous squamous cell carcinomas and responds to acute UV exposure in the epidermis of both humans and mice. Therefore, PD-L1 may represent both a target for precision prevention of cutaneous squamous cell carcinoma and biomarker of risk for this cancer. Taken together their data indicate that the stress response to UV may be linked to keratinocyte-derived expression of proteins typically studied in the context of inflammatory cells, providing avenues to novel topical interventions for NMSC prevention or treatment.

\section{Discussion on Session 1}

There was a fair amount of discussion regarding identification of appropriate animal models for prevention studies. A critical shortcoming still persists for the generation, characterization, and validation of models that represent high-risk cohorts and for the development of protocols to test potential preventive interventions in these models. In line with the discussion of model systems to understand the biology and testing immunoprevention therapies, there was discussion on what would be a good animal model system to test neoantigens as cancer prevention vaccine, especially if the frequency of neoantigens is low.

Some organ sites lack robust animal models that can recapitulate the genetics of human tumors. Differences in mouse strains also highlight that humans are genetically diverse species, so we need to account for differential responses to the same agent. Discussants pointed out the need for more validated genetically engineered mouse models, humanized mouse models, and the fact that no one tumor model necessarily appeared to be superior to other models for any given organ site. Possible use of multiple animal models using different strains or different species (mouse and rat, for example) and different initiators of tumorigenesis were discussed as well.

Another problem discussed was the heterogeneous and diverse genetics of the patient populations that are not cap- 
tured with relatively homogenous genetic animal models and that this should be factored into prevention studies. A focus on the degree of specific animal models similarities and dissimilarities with human patients with respect to cancer development and their immune systems needs to be factored into the experimental design of pre-clinical in vivo prevention studies. The need for a special workshop on animal models was suggested.

There was discussion on timing and duration of immunomodulatory agent administration to avoid unwanted side effects and toxicity. Panelists discussed if uncontrolled administration of immunomodulatory agents could lead to autoimmune like condition. Panelists suggested that there is a need for further understanding of the biology of tumor formation, immunological changes occurring during tumor progression, and the need to intercept the pre-neoplastic process at the appropriate stage(s). Some toxicity questions from the attendees included:

1. Are there any organized efforts to develop platforms for local delivery of immune modulators to avoid systemic toxicities?

2. In a prevention setting one could be treating patients with these agents for an extended period of time. What type of toxicities one would anticipate?

3. Is there a mechanism by which these agents could be specifically targeted to early developing tumor lesions?

Use of novel animal models and utilization of multiple models for immune analysis may help address toxicity issues. Panelists discussed the need to explore different delivery strategies to optimize the benefits to harm ratio. More research needs to be done on alternative delivery and sustained drug release strategies.

Suggestions that arose included on the possibility to include numerous antigens in the target vaccines, say, top $10 \%$ of recurrent mutations in drivers KRAS, BRAF etc., given the large capacity of RNA and adenovirus vaccines to include hundreds of neoantigens.

Other questions raised by the attendees that need further in-depth studies included:

1. Is a hierarchical order of epigenetic alterations during early to late colorectal tumorigenic process or in other cancer types normally observed?

2. Are there unique epigenetic changes known to be linked to specific oncogenes?

3. For immune recognition and immune memory development against preinvasive neoplasia, immune cells need to transit the basement membrane barrier twice; once to enter the tissue to surveil the epithelium and once to engage the immune system in order to promote development of the immune system. This raises the question of how much of a role does the permeability of the basement membrane play in the immune response to the developing tumor?

4. Would modulating this permeability be beneficial or too risky for prevention?

\section{SESSION 2: COMBINATIONS OF IMIMUNOMODULATORS WITH CANCER PREVENTIVE AGENTS OR VACCINES}

Dr. Johnson's talk "Treatment with Avasimibe, an ACAT Inhibitor, Enhances Tumor Vaccine Efficacy", described that AVA complements the efficacy of a multi-peptide Kras vaccine in preventing lung cancer development and growth. While treatment of cancer patients with vaccines has had limited success in the clinic, researchers have speculated that use of vaccines for cancer prevention might be able to achieve better efficacy. One approach to cancer immunoprevention has been to target mutant proteins that are known to drive oncogenesis. Dr. Johnson's lab has been investigating use of a multi-peptide vaccine targeted against both mutant and wild-type Kras for lung cancer prevention, and although the vaccine demonstrates preventive efficacy in preclinical animal models, there is still room for improvement.

Based on a recent study showing that the acyl coenzyme A:cholesterol acyltransferase 1 inhibitor (ACAT1), AVA, could potentiate CD8 T-cell anti-tumor responses by modulating cholesterol metabolism, it is hypothesized that treatment with AVA would improve the immunoprevention efficacy of the Kras vaccine. In support of this hypothesis, the combination of Kras vaccines and treatment with AVA enhanced the inhibition of lung tumor progression in a syngeneic Kras+ tumor cell implantation model as well as development of tumors in KrasLA1 transgenic mice. Tumor inhibition correlated with increased infiltration of functional CD8 T cells at tumor sites in conjunction with decreased frequencies of regulatory $T$ cells. Overall, these results suggest that this novel combined treatment approach could be used as immunoprevention for lung cancer.

Dr. Disis's presentation "Combination Chemo-Immunoprevention of Colon Cancer", described the potential of combinations of Th1 selective vaccine and NSAIDs for colon cancer treatment and prevention. The success of vaccines in the prevention of infections has resulted in immune targeting approaches being explored for the treatment and prevention of a variety of non-infectious diseases such as cancer. Recent evidence indicates that Type I immunity, associated with the production of IFN- $\gamma$, is needed for cancer eradication. Type I T-cells enhance cross priming at the site of cancer initiation by activating local antigen presenting cells to more efficiently present immunogenic proteins or tumor antigens to $\mathrm{T}$ cells. Cross priming is the primary method by which immunity is generated against cancer as tumor cells do not express the recognition molecules needed for immune activation. IFN- $\gamma$ is primarily secreted by CD4+ Th1 cells. Vaccine strategies designed to elicit tumor antigen specific Th1 immunity have the potential to generate epitope spreading (a broadening of immunity to additional antigens), concurrently stimulate anti- 
gen specific cytotoxic (cytotoxic T lymphocytes [CTL]) CD8+ T cells, and establish immunologic memory.

Immunologic memory will ensure that the destructive immune response will deploy when the antigen is expressed in the future. Although many vaccine strategies will equally generate both Type I and Type II T-cell responses against non-mutated tumor antigens, methods have been developed to construct Th1 selective vaccines which can generate high levels of unopposed Type I immune responses. The ability to generate Type I immunity without significant self-regulation has allowed the generation of multi-antigen cancer vaccines, targeting non-mutated proteins, to show improved efficacy and immunogenicity.

Studies from this group have demonstrated in mouse models that immunizing against multiple antigens is more effective clinically than immunizing against a single antigen. Most likely this is due to the higher levels of T-cells elicited with multi-antigen vaccines. In the therapeutic setting, multi-antigen Th1 selective vaccines are being used to prevent disease relapse in high-risk patients. This group suggested that vaccines could be developed for cancer interception by treating high risk lesions such as colonic adenomas. They used genomic approaches to identify putative antigens that are involved in initiating malignancy by identifying genes upregulated in both polyps and early-stage colon cancer. Th1 selective vaccination targeting these proteins was developed.

Vaccination could significantly inhibit the development of colonic tumors in chemically induced colon tumor models (azoxymethane model) and prevent the growth of intestinal polyps in the APC ${ }^{\min }$ mouse. Non-steroidal anti-inflammatory agents (NSAIDS) also inhibit polyp growth. They demonstrated that treatment of either of these models with Naproxen alone will result in some level of polyp control. Naproxen inhibited the expression of both PD-L1 and lymphocyte-activation gene 3 (LAG3) in the tumor cell via a COX-2 dependent mechanism. Combination immunoprevention with both the NSAID and vaccination resulted in superior control of polyp formation compared to vaccine or NSAID alone. Data were presented exploring the role of intermittent NSAID use and the timing of NSAID use in augmenting the efficacy of a multi-antigen vaccine designed for colon cancer interception.

Dr. Stanton's talk "Modulating the Immune Environment for Primary and Secondary Breast Cancer Prevention", focused on the combination of RXR agonists plus multi-antigen vaccines for the prevention of breast cancer. In breast cancer, increased immune infiltrate prior to neoadjuvant chemotherapy predicts improved pathologic complete response and improved survival; however, almost half of breast tumors have no CD8+ T cell infiltrate [13]. Therefore, discovering well tolerated agents that enhance the anti-tumor immune response in the tumor may improve response in breast cancer patients. The oral agent bexarotene (a retinoic receptor agonist) showed a $20 \%$ disease response as a single agent in metastatic breast cancer [14] and has been shown to increase CD8+ T cell tumor infiltration and decrease CD8+ $T$ cell apoptosis in vitro [15]. Evaluating RXR expression in human peripheral blood monocytes $(n=10)$, her laboratory found that RXR is not expressed in NK T-cells or B-cells and in only a minority of CD4+ $(5.1 \% \pm 4 \%)$ and CD $8+$ T-cells $(3.6 \% \pm 3 \%)$. RXR, however, was expressed in $24.9 \% \pm 13 \%$ of macrophages, $38.6 \% \pm 14 \%$ of plasmacytoid dendritic cells $(\mathrm{pDC})$, and $33.1 \% \pm 16 \%$ of monocyte-derived dendritic cells $(\mathrm{mDC})$.

Peripheral blood mononuclear cells treated with increasing doses of bexarotene for 48 hours had increased co-stimulatory CD40 expression on $\mathrm{mDC}(P=0.0011$ between 0 and 20 $\mu \mathrm{M}$ bexarotene) and increased type 1 cytokine release including IL-1 $\beta(P=0.04$ between 0 and $20 \mu \mathrm{M}$ bexarotene $)$ and TNF $\alpha$ ( $P=0.03$ between 0 and $20 \mu \mathrm{M}$ bexarotene) but not type 2 cytokine release of IL-10 and IL-4. This demonstrates that bexarotene can activate type 1 dendritic cells. They have since demonstrated similar results with 9cUAB30. Activated type 1 dendritic cells are most effective at presenting tumor antigens from dying tumor cells. Dr. Stanton's laboratory therefore wanted to determine if RXR agonists bexarotene and 9cUAB30 could enhance the effect of a multi-antigen polyepitope DNA vaccine against HER2-insulin-like growth factor-binding protein 2 [IGFBP2]-Insulin-like growth factor 1 receptor [IGF1R] in transgenic mouse mammary tumor models genetically and immunologically similar to human luminal B breast cancer (TgMMTV-neu) and triple negative breast cancer (C3(1)Tag) by enhancing the type 1 immune response in the tumor.

Treatment with $150 \mu \mathrm{g}$ HER2-IGFBP2-IGF1R vaccine increased antigen-specific IFN- $\gamma$ T cells, but not antigen-specific IL10 T cells, as compared to control vaccination with empty vector. Daily oral administration of $30 \mathrm{mg} / \mathrm{kg}$ bexarotene for 5 days prior to the HER2-IGFBP2-IGF1R vaccination series increased the IFN- $\gamma$ immune responses to HER2, IGFBP2, and IGF1R by 1.2, 2.4 and 2.2-fold, respectively, as compared to the HER2-IGFBP2-IGF1R vaccine alone. Daily administration of 200-mg/kg 9cUAB30 for 5 days prior to the HER2-IGFBP2-IGF1R vaccination series increased the IFN- $\gamma$ immune responses to HER2, IGFBP2, and IGF1R by 2.0, 2.3 and 1.7fold, respectively, as compared to the HER2-IGFBP2-IGF1R vaccine alone. Control vaccination with either $9 \mathrm{cUAB} 30$ or bexarotene had no impact on antigen-specific IFN- $\gamma \mathrm{T}$ cell response. Type I DCs are important for producing polyfunctional $\mathrm{CD} 4+\mathrm{T}$ cells that release not only IFN- $\gamma$ but also TNF- $\alpha$ and IL-2.

Polyfunctional $\mathrm{T}$ cells induce a longer lasting and more effective immune response in vaccines both for infectious diseases and cancer. Stanton's laboratory demonstrated that the addition of bexarotene or 9cUAB30 increased antigen-specific polyfunctional T cells in the TgMMTV-neu ( $\mathrm{n}=$ 15) transgenic mouse mammary tumor model while vaccination alone did not. There was an average of $1.3 \% \pm 0.2 \%$ antigen-specific CD4 polyfunctional T cells and $2.7 \% \pm 0.7 \%$ 
antigen-specific CD8 polyfunctional T cells with empty vector and vehicle control (sesame oil). HER2-IGFBP2-IGF1R vaccination following $30 \mathrm{mg} / \mathrm{kg}$ bexarotene treatment increased polyfunctional $\mathrm{T}$ cells to an average of $6.1 \% \pm 2.0 \%$ antigen-specific CD4 polyfunctional T cells $(P=0.07)$ and $20.3 \% \pm 4.1 \%$ antigen-specific CD8 polyfunctional T cells $(P$ $=0.0003)$. HER2-insulin-like growth factor-binding protein 2 (IGBP2)-IGF1R vaccination after $200 \mathrm{mg} / \mathrm{kg}$ 9cUAB30 increased antigen-specific polyfunctional $\mathrm{T}$ cells to $7.6 \% \pm$ $2.0 \%(P=0.01)$ and antigen-specific CD8 polyfunctional $\mathrm{T}$ cells to $17.6 \% \pm 4.1 \%(P=0.003)$. These data indicate that $\mathrm{RXR}$ agonists have an immunostimulatory role with multi-antigen cancer vaccines and may augment the anti-tumor activity of vaccines.

\section{Discussion on Session 2}

Discussion centered on optimization of dosing for combination prevention studies. How can we optimize the risk:benefit to minimize toxicities while enhancing efficacy? Since some of the toxicity is related to the peak exposure, different dosing regimens and dosing schedules need to be considered that could include slow-release formulations, intermittent dosing, and the use of a single high concentration bolus. These dosing schedules might improve responses while minimizing harms. Optimization of vaccine efficacy through dose optimization will be important as increased research in this area is resulting in the development of new potential preventive vaccines. Using different adjuvants or vectors to deliver the vaccines could also help as well as determining the optimal separation of booster doses.

There was a quite a bit of discussion on immunomodulatory effects of NSAIDs. A variety of cancer prevention agents such as NSAIDs and other targeted drugs have been shown to act as potential immunomodulatory agents. It is not clear how these immune-based mechanisms of action change the way we study and potentially utilize these agents for cancer prevention. Will there be different approaches that we need to take when evaluating these agents? Panelists suggested that the anti-proliferative effects of NSAIDs may be contributing to the immune response to the developing tumor and that the immunomodulatory effects of these agents need to be better defined.

Other questions raised by the attendees included:

1. How early are vaccine targets being overexpressed in the animal models and in the sporadic and FAP patients?

2. What should be the timing of vaccine administration?

3. What are the barriers to moving combination approaches from preclinical studies to the clinic?

4. How should combination clinical trials be conducted?

\section{SESSION 3: IMMUNE CHECKPOINT INHIBITORS IN IMMUNOPREVENTION}

Dr. Pollak's talk "Immunoprevention of Cancer in Muir Torre Syndrome with a Checkpoint Inhibitor: What are the Broader Implications of a Successful Case?", provided a case report of a man with Muir-Torre syndrome. Immune checkpoint blockade therapy provides substantial benefits for subsets of patients with advanced cancer, but its utility for cancer prevention is unknown. Lynch syndrome (MIM 120435) is characterized by defective DNA mismatch repair and predisposition to multiple cancers. A variant of Lynch syndrome, MuirTorre syndrome (MIM 158320), is characterized by frequent gastrointestinal tumors and hyperplastic or neoplastic skin tumors.

Pollak's group reported the case of a man with Muir-Torre syndrome who had 136 cutaneous or visceral hyperplastic or neoplastic lesions over a period of 19 years (mean 7.5 neoplasms/year, range 2 to 26 ) prior to receiving pembrolizumab immunotherapy as part of multi-modality treatment for invasive bladder cancer. He not only had a complete response of the bladder cancer, but also demonstrated an absence of new cancers during a 22-month follow-up period on pembrolizumab. Because of concern of risks of immunotoxicity, his pembrolizumab was discontinued when he was without evidence of any neoplastic disease. Six months later, he developed stomach cancer as well as further cutaneous lesions. Resumption of pembrolizumab resulted in regression of all lesions and prevention of appearance of new lesions. This case adds to the rationale for exploring the utility of immune checkpoint blockade for cancer prevention, particularly for patients with DNA repair deficits. Optimum dosing and timing in the context of prevention may differ from regimes used for cancer treatment.

Dr. Schlom's presentation "The Spectrum of Opportunities for the Immunoprevention of Carcinomas", described the challenges and opportunities for intercepting cancer development with immunoprevention strategies. There are enormous opportunities to employ various modes of modulating the immune system toward the prevention of various cancers, and intercepting cancer development in the "preneoplastic" or early stage of development. This has been made possible by enormous strides in our understanding of the complexities of the immune system and the neoplastic process, and the development of a spectrum of agents that have the ability to activate the anti-tumor immune response, potentiate that immunity, reduce or eliminate immunosuppressive entities, and render tumor cells more susceptible to immune-mediated lysis. These agents have been evaluated in preclinical studies, and clinical studies are ongoing employing these agents as monotherapy and in combination therapies in patients with a range of cancers. Some of the "preneoplastic" conditions that are now ripe for immune intervention are cervical intra-epithelial neoplasia 3, Lynch syndrome polyps, colon polyposis, 
Barrett's esophagus, oral epithelial dysplasia, and low-grade prostate cancer.

Dr. You's presentation "Potentiation of Multi-peptide Lung Cancer Vaccines with Immune Checkpoint Antagonist CA170", described that CA170 enhances the efficacy of the KRAS vaccine by increasing the effector CD4+ T cell response and decreasing immunosuppression from both myeloid-derived suppressor cells (MDSCs) and Regulatory $T$ cells (Tregs) in mouse models of lung cancer. Expressed on cells of the myeloid and lymphoid lineages, V-domain immunoglobulin suppressor of $T$ cell activation (VISTA) is an emerging target for cancer immunotherapy. Blocking VISTA activates both innate and adaptive immunity to eradicate tumors in mice. Using a tripeptide small molecule antagonist of VISTA, CA170, Dr. You's laboratory found that it exhibited potent anticancer efficacy on carcinogen-induced mouse lung tumorigenesis. Remarkably, lung tumor development was almost completely suppressed when CA170 was combined with an MHCll-directed KRAS peptide vaccine. Flow cytometry and single-cell RNA sequencing revealed that CA170 increased CD8+ T cell infiltration and enhanced their effector functions by decreasing the tumor infiltration of MDSCs and Tregs, while the KRAS vaccine primarily induced expansion of CD4+ effector T cells. VISTA antagonism by CA170 revealed strong efficacy against lung tumorigenesis with broad immunoregulatory functions that influence effector, memory and regulatory $\mathrm{T}$ cells, and drives an adaptive $\mathrm{T}$ cell tumor-specific immune response that enhances the efficacy of the KRAS vaccine.

Dr. Zhu's talk "Using mouse models to discover new immunotherapy approaches for liver cancer", discussed the presence of neoantigens in the cirrhotic liver that may be targetable with immune checkpoint inhibitor $(\mathrm{ICI})$ administered before tumorigenesis to prevent development of hepatocellular carcinoma (HCC). Patients with cirrhosis are in a highrisk state for the development of $\mathrm{HCC}$, therefore cirrhosis represents an opportunity for liver cancer prevention. Dr. Zhu's laboratory has shown that in cirrhotic tissues, there is an accumulation of mutations and neoantigens that may be specifically targetable with cancer immunotherapy. They have also shown that immune checkpoint inhibition with anti-PD1 therapies can prevent tumorigenesis in mouse models of HCC. Reductions in tumorigenesis were accompanied by $T$ cell infiltration into the liver. Importantly, anti-PD-1 therapy did not impair liver function or worsen overall liver health. Given the safety observed with long-term immunotherapy use in humans, an immunotherapy chemoprevention strategy could provide a high benefit to risk ratio in select patients. In this talk, Dr. Zhu discussed immunotherapy in cancer prevention, potential opportunities for patient selection, and new ways to discover immunotherapy combinations for both prevention and treatment.

\section{Discussion on Session 3}

A major focus of the discussion was whether immune checkpoint inhibitors can be used in the prevention setting to reduce the mutation burden following exposure to carcinogenic chemicals. ICls by themselves may not be sufficient as there could be changes in tumor antigen levels as a result of certain exposures or in certain tumor types. Further discussions include the oral bioavailability, relatively short half-life, and low systemic toxicity of small molecule ICls are some of the advantages over monoclonal antibodies.

Panelists discussed the potency of small molecule $\mathrm{ICl}$ compared to anit-PD-1 treatment and the toxicity issues that may occur when ICls are administered as a single bolus dose compared to the standard course of treatment. Another question was whether one could reduce the mutational burden in the older population by treating older individuals at as yet undefined age(s). A key question is how to identify what types of mutations may be susceptible or resistant to ICI treatment. It was pointed out that there are a lot of variables to consider that include tumor characteristics and patient-specific heterogeneity. It is unclear how long treatment with ICls should be done in this setting and how the treatment can be tailored to different at-risk populations such as the elderly population.

\section{ACKNOWLEDGMENTS}

The workshop organizers Drs. Miller and Mohammed would like to thank our Staff Assistant, Ms. Wosene Asefa, for her outstanding assistance in the organization of the workshop. Drs. Wondrak and Dickinson were supported by $\mathrm{NIH} / \mathrm{NCl}$ P01CA229112, R03CA230830.

\section{CONFLICTS OF INTEREST}

Opinions expressed by the authors are their own and this material should not be interpreted as representing the official viewpoint of the U.S. Department of Health and Human Services, the National Institutes of Health, or the National Cancer Institute.

Drs. Dashwood, Dickinson, Disis, Jaffee, Khleif, Miller, Mohammed, Pollak, Schlom, Shoemaker, Wondrak, You and Zhu have no FCOls to declare. Dr. Johnson received support from Miltenyi Biotec. Dr. Stanton received research support through her institute from IMV Therapeutics.

\section{ORCID}

Altaf Mohammed, https://orcid.org/0000-0003-1058-6909

Roderick H. Dashwood, https://orcid.org/0000-0003-0351-4034 Sally Dickinson, https://orcid.org/0000-0002-2049-543X Mary L. Disis, https://orcid.org/0000-0001-7653-4648 Elizabeth M. Jaffee, https://orcid.org/0000-0003-3841-6549 Bryon D. Johnson, https://orcid.org/0000-0001-7416-3396

Samir N. Khleif, https://orcid.org/0000-0003-2707-5224 
Michael N. Pollak, https://orcid.org/0000-0003-3047-0604

Jeffrey Schlom, https://orcid.org/0000-0001-7932-4072

Robert H. Shoemaker, https://orcid.org/0000-0003-3608-1714

Sasha E. Stanton, https://orcid.org/0000-0002-4300-551X

Georg T. Wondrak, https://orcid.org/0000-0003-4799-8608

Ming You, https://orcid.org/0000-0002-9741-268X

Hao Zhu, https://orcid.org/0000-0002-8417-9698

Mark Steven Miller, https://orcid.org/0000-0003-1191-0977

\section{REFERENCES}

1. Keenan BP, Saenger Y, Kafrouni MI, Leubner A, Lauer P, Maitra A, et al. A Listeria vaccine and depletion of T-regulatory cells activate immunity against early stage pancreatic intraepithelial neoplasms and prolong survival of mice. Gastroenterology 2014;146:1784-94.e6.

2. Chu NJ, Anders RA, Fertig EJ, Cao M, Hopkins AC, Keenan $\mathrm{BP}$, et al. Inhibition of miR-21 regulates mutant KRAS effector pathways and intercepts pancreatic ductal adenocarcinoma development. Cancer Prev Res (Phila) 2020;13:569-82.

3. Zdanov S, Mandapathil M, Abu Eid R, Adamson-Fadeyi S, Wilson W, Qian J, et al. Mutant KRAS conversion of conventional T cells into regulatory T cells. Cancer Immunol Res 2016;4:35465.

4. Spranger S, Bao R, Gajewski TF. Melanoma-intrinsic $\beta$-catenin signalling prevents anti-tumour immunity. Nature 2015;523:2315.

5. Zelenay S, van der Veen AG, Böttcher JP, Snelgrove KJ, Rogers $\mathrm{N}$, Acton SE, et al. Cyclooxygenase-dependent tumor growth through evasion of immunity. Cell 2015;162:1257-70.

6. Xue W, Zender L, Miething C, Dickins RA, Hernando E, Krizhanovsky $\mathrm{V}$, et al. Senescence and tumour clearance is triggered by p53 restoration in murine liver carcinomas. Nature 2007;445:656-60.

7. Peng W, Chen JQ, Liu C, Malu S, Creasy C, Tetzlaff MT, et al. Loss of PTEN promotes resistance to $T$ cell-mediated immunotherapy. Cancer Discov 2016;6:202-16.

8. Chang K, Taggart MW, Reyes-Uribe L, Borras E, Riquelme E, Barnett RM, et al. Immune profiling of premalignant lesions in patients with Lynch syndrome. JAMA Oncol 2018;4:1085-92.

9. Llosa NJ, Cruise M, Tam A, Wicks EC, Hechenbleikner EM, Taube JM, et al. The vigorous immune microenvironment of microsatellite instable colon cancer is balanced by multiple counter-inhibitory checkpoints. Cancer Discov 2015;5:43-51.

10. Kobayashi KS, van den Elsen PJ. NLRC5: a key regulator of MHC class I-dependent immune responses. Nat Rev Immunol 2012;12:813-20.

11. Rajendran P, Johnson G, Li L, Chen YS, Dashwood M, Nguyen $\mathrm{N}$, et al. Acetylation of CCAR2 establishes a BET/BRD9 acetyl switch in response to combined deacetylase and bromodomain inhibition. Cancer Res 2019;79:918-27.

12. Kapoor S, Gustafson T, Zhang M, Chen YS, Li J, Nguyen N, et al. Deacetylase plus bromodomain inhibition downregulates ERCC2 and suppresses the growth of metastatic colon cancer cells. Cancers (Basel) 2021;13:1438.

13. Stanton SE, Disis ML. Clinical significance of tumor-infiltrating lymphocytes in breast cancer. J Immunother Cancer 2016;4:59.

14. Esteva FJ, Glaspy J, Baidas S, Laufman L, Hutchins L, Dickler $\mathrm{M}$, et al. Multicenter phase II study of oral bexarotene for patients with metastatic breast cancer. J Clin Oncol 2003;21:999-1006.

15. Marzbani E, Inatsuka C, Lu H, Disis ML. The invisible arm of immunity in common cancer chemoprevention agents. Cancer Prev Res (Phila) 2013;6:764-73. 